\title{
Influence of dispersed fillers on properties and processability of polyvinyl chloride compositions
}

\author{
Ruslan Galeev ${ }^{1, *}$, Rashid Nizamov ${ }^{2}$, and Layla Abdrakhmanova ${ }^{2}$ \\ ${ }^{1}$ Kazan Federal University, 423812, 68/19 , 335, Naberezhnye Chelny, Russia \\ ${ }^{2}$ Kazan state University of architecture and construction, 420043, Zelenaya St. 1, Kazan, Russia
}

\begin{abstract}
In this reaserch, the results of filling PVC compositions are presented on the example of only two types of dispersed fillers from among industrial wastes, presented in the form of fine particles and highly porous grains:beghouse dust and expanded perlite sand. The influence of the type of filler on the value of mechanical strength was also studied by the method of measuring it under one-side pressure. It was established that when filling with ultrafine filler BP, an increase in the elastic modulus and complete "degeneration" of the forced high elasticity are observed. It seems interesting the change of the melt flow index of PVC compositions with the introduction of both fillers.
\end{abstract}

\section{Introduction}

Polyvinyl chloride (PVC) has wide possibilities for modification (plasticization, filling, combination with other polymers), which are embedded in its chemical structure and the nature of the supramolecular structure [1,2]. But the specificity of the chemical structure and the nature of the supramolecular structure determine its serious disadvantages - high viscosity of the melts during processing, low resistance to mechanothermal influences and low impact strength of hard materials. Dispersed fillers of various nature, widely used for filling PVC compositions, in general, increase the viscosity of melts and the temperature of polymer processing [3-5]. This leads to a decrease in the thermal stability of the compositions and to a possible deterioration in the mechanical properties of filled composites. That is why, when choosing fillers, it is necessary to take into account simultaneously the effect of fillers on processability and on the final functional parameters of materials.

The formation of boundary layers during PVC filling is associated not only with a change in the supramolecular structure of the polymer, mobility, packing density, the appearance of orientational effects, but also with the chemical structure of the

\footnotetext{
* Corresponding author: grr1981@outlook.com
} 
polymer [6-9]. All technological and operational indicators are determined by the conditions for the formation of boundary layers during polymer-filler interaction [10], which, in turn, is determined in addition to the properties of the polymer matrix and all the basic physical and chemical characteristics of the fillers, such as the specific surface area, chemical and mineral compositions etc.

\section{Experimental part}

When choosing fillers from among industrial industrial wastes for plasticized and rigid PVC compositions, the main emphasis is on the presence of components that give them specific properties for a multifunctional effect on the matrix. Particularly important is the influence of morphology and particle size, on which the degree of filling and processing conditions of polymer compositions depends.

In this reaserch, the results of filling PVC compositions are presented on the example of only two types of dispersed fillers from among industrial wastes, presented in the form of fine particles and highly porous grains:

1 - "beghouse dust" (BD), characterized by a bimodal particle size distribution and an average particle size of 0.7 and 25 microns;

2 - expanded perlite sand (EPS) with an average particle size of 30 microns, but for which there is a large dispersion of particle sizes (from 0.1 to 250 microns).

Expanded perlite sand in the form of spent heat filling the cryogenic units of Nizhnekamskneftekhim is in dumps.

"Behousehouse dust" is characterized by a bimodal particle size distribution and an average particle size of 0.7 and 25 microns, and expanded perlite sand has an average particle size of 30 microns, but it has a large particle size dispersion (from 0.1 to 250 microns) (fig. 1 ).
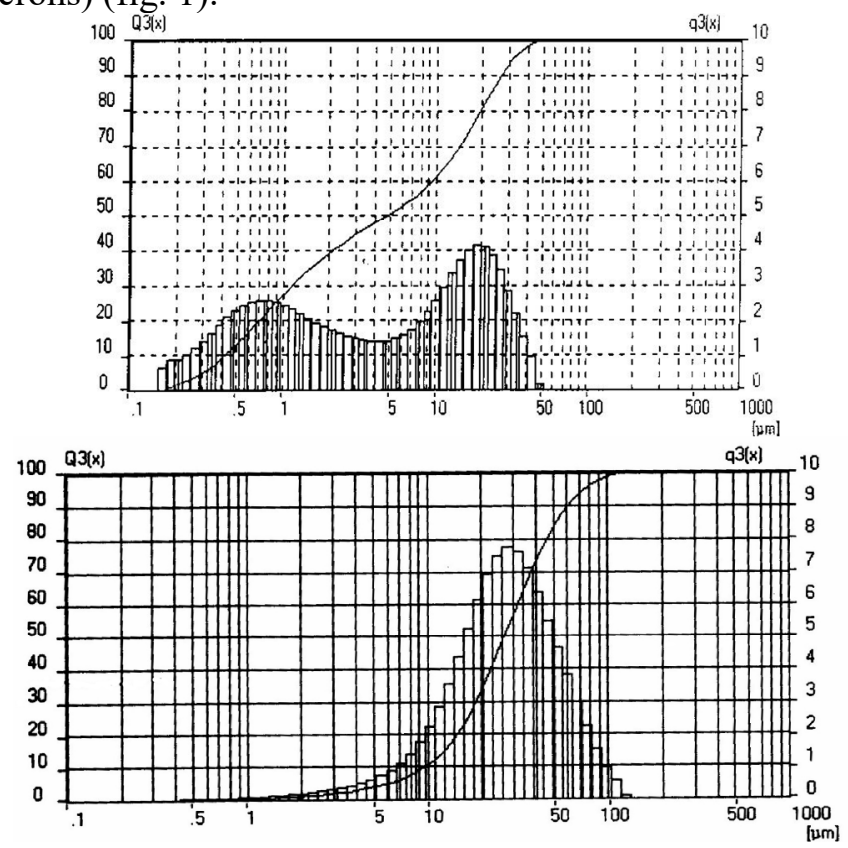

Fig. 1. Integral and differential curves of particle size distribution of a) behouse house dust; $b$ ) expanded perlite sand. 


\section{Discussion}

In the case of $\mathrm{BD}$, the presence of bimodality in the distribution of particles and the specific chemical nature of their surface causes the formation of aggregates. Aggregates are formed from particles of different sizes, which practically minimizes the free porous volume in the aggregates (Fig. 2a). The size of the EPS particles is approximately equal to the size of the aggregates of particles of the beghouse dust (Fig. 2b).

However, it can be assumed that during the processing of PVC compositions during the implementation of shear deformations, BD aggregates will be destroyed, the strength of which is low, as well as fragile porous EPS particles. With the introduction of the same volumes of BD and EPS (bulk density of BD is higher than that of EPS more than 20 times), the number of particles will be significantly larger in $\mathrm{BD}$.

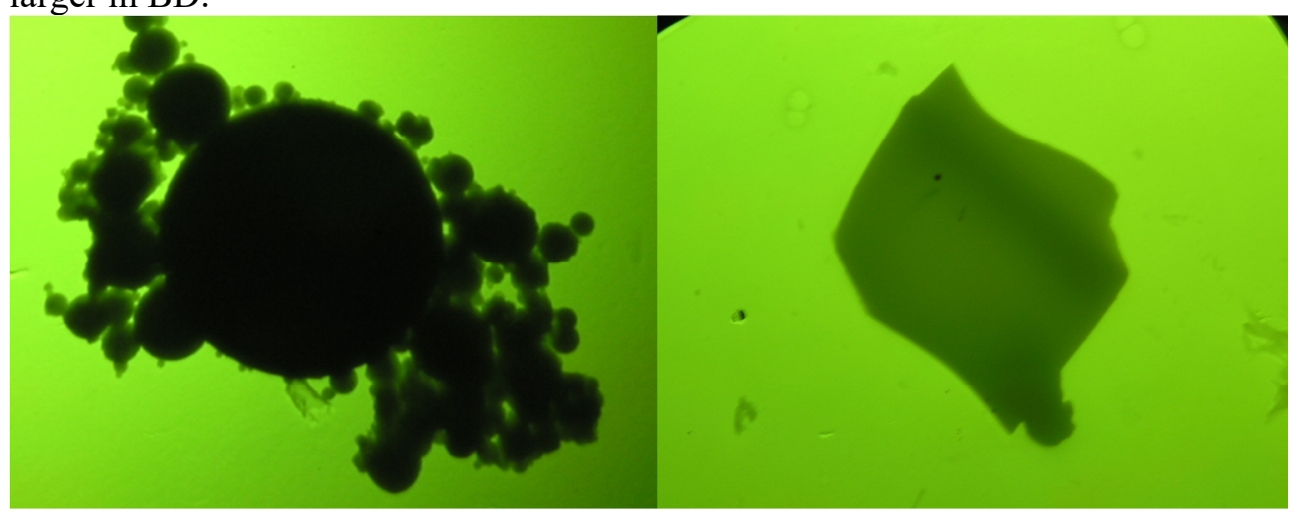

Fig. 2. Microphotographs of particles of a) beghouse dust; b) expanded perlite sand.

Both fillers are inert fillers. However, the strength of PVC films is higher with the introduction of $\mathrm{BD}$, which is probably due to the larger particle sizes of the EPS and the practically absence of particles with sizes less than 1 micron, as in BD.

The influence of the type of filler on the value of mechanical strength was also studied by the method of measuring it under one-side pressure. It was established that when filling with ultrafine filler BP, an increase in the elastic modulus and complete "degeneration" of the forced high elasticity are observed [11]. These changes are confirmed by microphotographs of samples obtained at the time of destruction (Fig. 3).

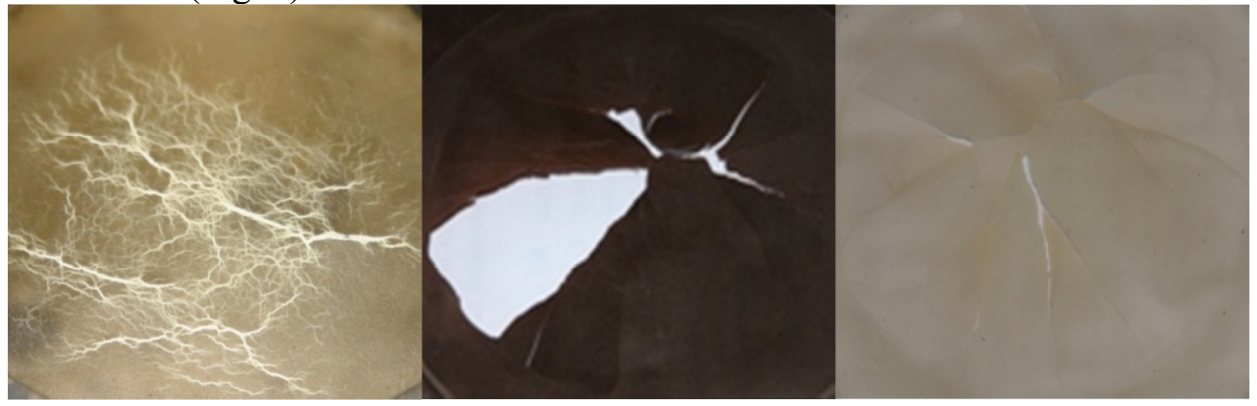

Fig. 3. Photographs of films at the time of destruction a) without filler; b) with beghouse dust; c) with expanded perlite sand. 
It seems interesting the change of the melt flow index of PVC compositions with the introduction of BD and EPS. As an example, Fig. 4 shows the concentration dependences of the melt flow index for plasticized compositions. In both cases, there is an increase of the melt flow index. In the case of BD filling, there is a sharper increase in melt flow index. This can be explained by a higher dispersion of BD. It should also be taken into account that the EPS, having a porous structure, sorb the plasticizer to a greater extent, thereby reducing its functional efficiency.

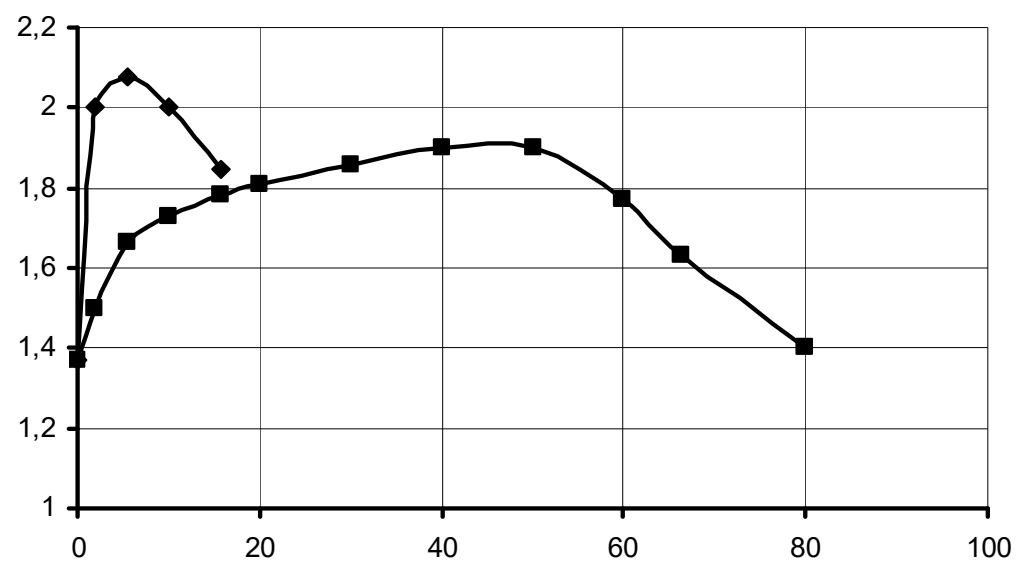

Fig. 4. The dependence of the melt flow index of the PVC composition on the content of beghouse dust (1) and expanded perlite sand (2).

These fillers also contribute to the thermostability of PVC compositions. BD significantly (approximately 3.5 times) increases the thermal stability of PVC, EPS affects this indicator to a much lesser extent. At first glance, this fact seems illogical, since the porous structure of perlite suggests its high sorption ability [12, 13], including hydrogen chloride released during the destruction of PVC. But if we assume that in some cells of the perlite particles DOP is sorbed, and also bear in mind the more developed surface of $\mathrm{BD}$ particles due to the high dispersion, then the data obtained are quite explainable.

The porous structure of ESP particles determines the physical mechanism of polymer stabilization due to the sorption of hydrogen chloride and the leveling of its autocatalytic effect on the destruction of the polymer. The presence of iron oxides usually has a negative effect on thermal stability [14, 15]; therefore, ESP, which is mainly represented by them, is effective only at concentrations not higher than $5 \%$, and then thermal stability decreases sharply. Iron oxides are present in very small amounts in the EPS composition, which does not accelerate the destructive processes of the polymer even with high filling. The fact that the effects of increasing thermal stability in absolute terms for EPS is not so high is obviously due to the fact that the mineral composition of the runway does not have a high reactivity with respect to hydrogen chloride.

In general, it should be emphasized that the optimal concentrations for BD is observed at concentrations much lower than for EPS. 


\section{Conclusion}

Thus, the considered fillers can be used in the formulation of PVC compositions instead of traditional fillers. Processability in the presence of BD and EPS is greatly facilitated. The stabilizing effect is due to the mechanism of chemical and physical stabilization, and changes in the mechanical properties are determined by the formation of interfacial layers at the polymer-filler interface and their structural features.

The quantitative relationships we have established between the properties of PVC compositions and the physicochemical properties of fillers can be used to a first approximation to predict the effectiveness of different types of inorganic fillers.

\section{References}

1. J. Summers, C. Daniels, Polyvinyl chloride (St. Petersburg, Profession, 2007)

2. M.W. Allsopp, Pure and Applied Chemistry 53(2), 449-466 (1981).

3. V.V. Guzeev, Polymer materials 7-8, 38-48 (2010).

4. M. Xanthos Functional fillers for plastics (St. Petersburg, Scientific Foundations and Technologies, 2010).

5. Zeich J. 7th Int. Plastic-Window Congress (Wuerzburg, Germany, 2009).

6. T.S. Ghong, E.B. Christiansen, A.D. Baer, J.Appl.Polym.Sci. 15, 2007-2021 (1971).

7. M. L. Kerber, A. M. Bukanov, S. I. Wolfson, I. Yu. Gorbunova, L. B. Kandyrin, A. G. Orphan, M. A. Sheryshev Physical and chemical processes in the processing of polymers (SPb, Publishing house NOT, 2013)

8. R.K. Nizamov, L.A. Abdrakhmanova, V.G. Khozin, Building materials based on polyvinyl chloride and multifunctional industrial waste (2008).

9. E.Kh. Karimov, O.Kh. Karimov, R.R. Daminev, E.M. Movsumzade, G.F. Sabitova, A.Yu. Sharipova, M.Yu. Cherezov, Electronic scientific journal Oil and Gas Business 5, 140-151 (2016).

10. O.F. Pozdnyakov, A.O. Pozdnyakov, V.R. Regel, Solid State Physics. 47(5), 924-930 (2005)

11. R.R. Galeev, S.N. Yakupov, R.I. Shafigullin, L.A. Abdrakhmanova, N.M. Yakupov, Abstracts X1Y of the All-Russian Conference "Structure and Dynamics of Molecular Systems" Kazan-Moscow Yoshkar-Ola-Ufa 65 (2007)

12. L.A. Abdrakhmanova, I.V. Kolesnikova, R.K. Nizamov, E.V. Khozina, Theses AllRussian. conf. "The structure and dynamics of molecular systems" $\mathbf{3}$ (Yoshkar-Ola, 2004)

13. S.R. Ivanova, K.S. Minsker, E.I. Nagumanova, R.K. Nizamov, S. Kazaryans, Plastics 12, 39-42 (2005).

14. Minsker K.S. Zaikov G.E. Plast. Masses 4, 27-35 (2001).

15. M. Schiller, B. Petzl, GAK 64, 369 (2011). 